\title{
Binomio y binómino, la confluencia de dos nombres en textos ma- temáticos renacentistas: algunas consideraciones etimológicas so- bre la designación de las expresiones algebraicas
}

\author{
ITZIAR MOLINA SANGÜESA \\ Universidad de Salamanca
}

\section{PRESENTACIÓN}

El objetivo de este trabajo pretende esclarecer algunas cuestiones relacionadas con la interpretación etimológica que aparece en varios repertorios lexicográficos - tanto de lengua española como de otras lenguas romances - sobre un conjunto de expresiones algebraicas.

Tras haber constatado entre los tratados matemáticos pertenecientes al corpus del Diccionario de la Ciencia y de la Técnica del Renacimiento (DICTER) ${ }^{1}$ la fluctuación del uso de dos nombres: binomio y binómino, para la designación de una de las más comunes y frecuentes expresiones algebraicas - la que se compone de dos términos-, comenzamos a investigar las cuestiones que en las siguientes líneas expondremos.

Estas designaciones aparecen, casi de manera exclusiva, en tres textos cuyos contenidos van de la aritmética al álgebra ${ }^{2}$, concretamente, en los capítulos diez, once y doce $^{3}$ del Libro primero de Arithmética algebrática (1552) de Marco Aurel ${ }^{4}$, en el libro

*El desarrollo de esta investigación ha sido posible gracias a la ayuda predoctoral (FPU), concedida en 2011 por el Ministerio de Educación, Cultura y Deporte (Ref.: AP2010-3663). Este trabajo se inserta en el marco del proyecto I+D+i: «El Diccionario de la Ciencia y de la Técnica del Renacimiento (DICTER): implantación definitiva en la Red» (Ref.: FFI2013-41386-P) financiado por la Dirección General de Investigación del Ministerio de Economía y Competitividad.

${ }^{1}$ Véase $<$ http://dicter.usal.es $>$.

${ }^{2}$ Uno de los aspectos más relevantes en la difusión y desarrollo de esta disciplina es que -a diferencia de las obras de Aurel (1552) y Pérez de Moya (1562), en las que el Álgebra es considerada como una variante elevada o un capítulo más de la Aritmética- en la obra del matemático portugués Pedro Núñez (1567), publicada 30 años antes en su lengua materna, asistimos a lo que Massa Esteve denomina como «la algebrització de les matemàtiques», esto es, «quan l'àlgebra comença a ser considerada una disciplina independent dins de la matemàtica» (2010: 101), ya que «al dedicar las dos primeras partes al Álgebra como tal confiere este a saber una entidad propia, que hasta entonces no se le había concedido» (Flórez Miguel 2006: 418).

Capítulo décimo. Trata de binóminos y residuos, y de sus definiciones, declaración y operación / Capítulo $11^{\circ}$. Trata de las definiciones de las raýzes quadradas de los binóminos, y de cómo se ha de sacar la raýz quadrada de los dichos binóminos / Capitulo 12. Trata de la raýz cúbica de los binóminos, y de sus definiciones y extractión (fols. 55v-fol. 69r). 
séptimo ${ }^{5}$ de la Arithmética práctica y speculativa (1562) de Juan Pérez de Moya ${ }^{6}$ y a lo largo del capítulo quince ${ }^{7}$ del Libro de Álgebra en Arithmética y Geometría (1567) de Pedro Núñez Salaciense ${ }^{8}$.

\section{ANÁLISIS}

A continuación, revisaremos y analizaremos, por un lado, el tratamiento etimológico de los términos algebraicos binomio y binómino, principalmente, y el de sus derivados: binómico y binominal. Por otro lado, comprobaremos la presencia de los mismos en el corpus textual del DICTER y la alternancia de uso de las formas acabadas en -nomio frente a -nómino. Asimismo, pasaremos revista a otras formaciones similares que designan expresiones algebraicas compuestas por más de dos términos, de las que, finalmente, extraeremos unas conclusiones.

\subsection{Binomio}

La etimología de este sustantivo es una cuestión bastante controvertida, motivo por el que presenta diversas interpretaciones en los distintos repertorios lexicográficos analizados. Por un lado, la penúltima edición del diccionario académico, el DRAE, y en las sucesivas ediciones del mismo desde $1884^{9}$, afirma que este término algebraico proviene de la unión de bi- y el gr. vouós 'parte, porción'.

De manera análoga a los repertorios lexicográficos de la Real Academia Española, hemos comprobado que varios diccionarios de lengua portuguesa, como el Dicionário da Língua Portuguesa (DLP) de Porto Editora o el Novo Dicionário da Língua Portuguesa (NDLP) de Cândido de Figueiredo, presentan la misma interpretación etimológica, es decir, consideran que binomio proviene «do latim BI-, 'duas vezes' + grego NÓMOS, 'porção; divisão'» (DLP: s. v.).

Por otro lado, el DECH postula una etimología distinta, al afirmar que binomio es un cultismo tomado del b. lat. BINOMIUM 'íd.', sustantivación del adjetivo BINOMIUS 'de dos nombres', y éste derivado del fr. NOM o del it. NOME 'nombre', procedentes del lat. NOMEN 'íd.'.

${ }^{4}$ Se conocen pocos datos sobre su biografia; de origen alemán, afincado en Valencia, fue maestro de escuela (1541) y publicó la primera obra impresa en España cuyos contenidos versan sobre la regla de la cosa o Álgebra, de ahí que en el prólogo exponga (1552: IIIr): «es cosa nueva lo que trato y jamás vista ni declarada, y podrá ser que ni aun entendida ni imprimida en España» (cf. Picatoste 1891; Rey Pastor 1926; Paradis y Malet 1989).

${ }^{5}$ En el que se enseña a sumar, restar, multiplicar y partir binomios (pp. 525-540), además de cómo obtener la raíz cuadrada y cúbica de los mismos (pp. 524-534) a lo largo de los artículos que componen el Capítulo nono. Trata del binomio y disjuncto.

${ }^{6}$ Matemático andaluz (Santisteban del Puerto, ca. 1513-Granada, 1597), fue uno de los autores más célebres del panorama científico del siglo XVI hispano. Su obra alcanzó multitud de ediciones (unas 30, desde la fecha de su primera publicación en 1562 hasta 1875) y fue muy conocida dentro y fuera de nuestras fronteras (cf. Picatoste 1891; Rey Pastor 1926; Leal y Leal 1971-1972; Valladares Reguero 1997).

${ }^{7}$ Capítulo 15. De las raizes de los binomios (fols. 112r-125v).

${ }^{8}$ Matemático portugués (Alcácer do Sal, 1502-Coimbra, 1578) es, junto a Pérez de Moya, uno de los autores más destacados del Quinientos (cf. Picatoste 1891; Rey Pastor 1926; Ventura Sousa 1985). $16 / 07 / 2013]$

Véase el Mapa de Diccionarios Académicos de la RAE: <http://web.frl.es/ntllet> [consulta: 
En esta misma línea, el TLFi corrobora que la hipótesis «plus vraisemblable est celle d'une adaptation du lat. médiév. BINOMIUM "quantité algébrique à 2 termes"» $\mathrm{y}$, del mismo modo que el $\mathrm{DECH}^{10}$, indica que se trata de un «terme employé par Gérard de Crémone (1114-1187) ${ }^{11}$, auteur d'une traduction latine des commentaires arabes de Fadl ben Hatim an-Naizîrî ${ }^{12}$ sur les dix premiers livres de la Géométrie d'Euclide», concretamente, del siguiente fragmento de los Anaritii in decem libros priores Elementorum Euclidis commentarii:

Quod ideo est, quoniam unumquodque sex binomiorum, cum dividuntur in duas lineas, quae sunt nomina ipsius, ipse [sic] continent superficiem medialem [ed. M. Curtze 1899: 246].

A continuación, postula que BINOMIUM «serait donc plutôt composé du lat. BIS et NOMEN» (TLFi: s. v.). No obstante, plantea otra hipótesis o alternativa posible: la que defiende el DRAE en su vigesimosegunda edición, es decir, que se trate de «une formation à partir du préf. BI-* et du gr. vouós 'part, portion'», pero puntualiza que «cette signification représente le sens premier du terme, seulement attesté au sens de 'province, district'». Por lo tanto, que el «terme de mathématiques serait une transposition au domaine scientifique de l'adj. lat. médiév. BINOMIUS '(d'une pers.) qui a 2 noms ${ }^{13}$, lui même latinisation des représentants romans du lat. NOMEN»; detalles que el DECH también tiene en cuenta al admitir que posteriormente se ha creído por error que bino-

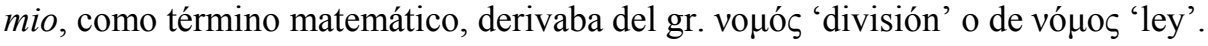

Del mismo modo, el VLI considera que este término matemático proviene «dal lat.

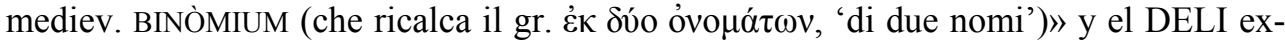
pone que binomio es un nombre tomado del «lat. BINÒMIUS comp. di BIS- 'due volte' e NOMEN 'nome', a cui altri sostituisce il gr. nomos 'legge, regola'»' 14 .

De acuerdo con esta última interpretación, hallamos que, en algunos tratados matemáticos del Quinientos, como la Arithmética redactada por Pérez de Moya, parece confirmarse que binomio se trata de un latinismo:

De la composición y origen del binomio: Los mathemáticos inventaron 15 quantidades acerca de las quales emplearon principal estudio [...]. De las 12 quantidades que diximos irracionales compuestas, las 6 son raýzes de números compuestos de

\footnotetext{
${ }^{10}$ Este sustantivo latino lo empleó por primera vez Gerardo de Cremona en el siglo XII para

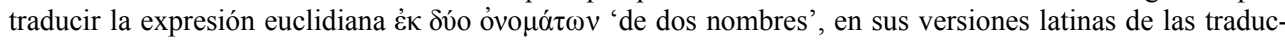
ciones arábigas de los matemáticos griegos (DECH: s. v. binomio).

${ }^{11}$ Italiano de nacimiento (Cremona, 1114-Toledo, 1187), fue uno de los personajes más ilustres y prolíficos de la Escuela de Traductores de Toledo. A pesar de que se conserva poca información acerca de la vida de este autor (cf. Boncompagni 1851) se sabe, sin embargo, que conocía el griego, el árabe y el latín, y que sus traducciones abarcan una multiplicidad de temas (tales como la astronomía, geometría, óptica, matemáticas o la alquimia).

${ }^{12}$ También citado como Al-Fadl b. Hätim Al-Nayrizi o al-Naiyritī (ca. 897-922), fue un astrónomo y matemático distinguido. Como matemático demostró el teorema de Pitágoras y realizó unos comentarios a los Elementos de Euclides, que fueron traducidos al latín por Gerardo de Cremona; sobre la traducción de los mismos, léase Vernet (1978: 120-122 y 2006²:178-182).

${ }^{13}$ «El adjetivo binomius existía ya, sin significado matemático, desde el siglo XI por lo menos, y de él procede el adjetivo cast. binomio 'de dos nombres', empleado por APal.» (DECH: s. v. binomio).

${ }^{14}$ Este término también aparece documentado, pero sin precisar su etimología, en el DLI y en el DCVB.
} 
dos quantidades, de do toman denominación los binomios, de bis y nomen, que quiere dezir cosa de dos nombres (Pérez de Moya 1562: 525).

En el corpus del DICTER aparece este término en 325 ocurrencias a lo largo de cuatro de sus textos matemáticos, aunque con suma frecuencia en los tratados de Pérez de Moya (85 ocurrencias) y Núñez Salaciense (184 ocurrencias):

Multiplico el uno por el otro, y vernán $2+\sqrt{ } 3$. Nota que el binomio o residuo, en tal caso, tomarás por sola una quantidad simple, multilicando $\sqrt{u}$. de $11 \sqrt{2}$ con $\sqrt{u}$. de $11+\sqrt{ } 2$, y vernán $11+\sqrt{ } 2$. Y luego multiplico -3 con +3 , y vernán -9 . Éstos quito de $11+\sqrt{2}$, y quedarán $2+\sqrt{3}$, que en multiplicar en cruz se va el + por el -, siendo un binomio con su residuo multiplicado, como en su lugar has visto (Aurel 1552: fol. 112r).

Como se collige del artículo primero, los binomios se causan de un ajuntamiento de una cosa differente con otra. Assí como si quisiesses summar 4 con r.7, en tal caso porque r.7 no tiene raýz racional juntarás lo uno con lo otro con la dictión del p., diziendo que monta 4 p. r.7, y queda hecho un binomio (Pérez de Moya 1562: 529).

Pero para multiplicar un binomio por el su residuo, no ay necesidad de tanta obra, porque las dos multiplicaciones que se hazen en cruz ninguna cosa montan, porque una deshaze la otra. Por esta causa, bastará summar los quadrados de las partes y el uno d'ellos es declarado por más y el otro por menos (Núñez 1567: fol. 50r).

Presenta, binomio, una única ocurrencia en los Descubrimientos geométricos (1598: fol. $17 \mathrm{v}$ ) de Joan Alfonso de Molina $\mathrm{Cano}^{15}$, quien alude a la herencia euclídea del Álgebra:

Y no me ha parescido hazer más d'estas 50 partes, por ser impossible que a sus tan menudas divisiones las pueda conprehender la vista y estar satisfecho que, por sorda que el adversario ponga qualquiera linia, son ellas suficientes para darle con el compás su valor discreto tan preciso, como si se le pronunciasse después de aver sacado d'ella por números su raýz quadra cúbica, o de otra qualquier dignidad, y aun mucho mejor (si dezir se puede), por no estar estas mis divisiones sujetas a hierrazos de pluma, ni a essaminar muy menudamente si se sacó bien la raýz del binomio o residuo, como muestra la álgebra sustancia del 2 y 10 libro de Euclides, de tan poquitos bien entendida,

de la que reconoce las malas interpretaciones e incomprensiones.

\subsubsection{Binómico}

Este adjetivo relacional derivado de binomio no se documenta en ninguno de los diccionarios consultados, a excepción del portugués DLP $^{16}$; de ahí se puede explicar el

${ }^{15}$ Afamado geómetra del Quinientos (cf. Rey Pastor 1926: 134-136) fue autor de manuales sobre Matemáticas y Geometría ya que, como afirma Picatoste, «su buen juicio le hizo comprender la necesidad del estudio de las Matemáticas para todos los ciudadanos» (1891: 196). De ahí que en el prólogo de su obra exponga: «porque es muy necessaria a toda qualidad de personas esta admirable disciplina, me atrevería a aconsejar a los que mandan y goviernan repúblicas, que entretengan en ellas personas doctas, para que públicamente la lean y enseñen, y a persuadir a los padres, a quien Dios dio hijos y possibilidad para bien doctrinallos, a que, en acabando de saber leer, escrivir y contar, los metan en ella, assegurándoles que, quando d'ella no saquen otro fructo que el averse acostumbrado a tratar con verdades que con otras verdades se compruevan» (1598: IIv).

${ }^{16}$ De binomio + -ico. Sobre el sufijo -ico véase Pharies (2002: 306-308). 
hecho de que se constate en 19 ocurrencias en nuestro corpus exclusivamente en el Álgebra del matemático portugués Pedro Núñez Salaciense.

Se podría definir como lo «perteneciente o relativo al binomio» (DRAE ${ }^{22}: s . v$.), tal y como se deduce de los ejemplos hallados:

La regla para hallar las raízes cubas binómicas de los binomios cubos traeremos por la misma manera y por los mismos discursos que la hallamos. Y la invención no es differente del arte que tuvimos para sacar las raízes quadradas de los binomios, puesto que todo binomio es quadrado, pero no es cubo qualquier binomio (Núñez 1567: fol. 114v)

Cumple, luego, inquirir si el dicho binomio raíz de $251 / 27$ más 5 es cubo, y esto haremos buscándole la raíz cuba por entrambos los nombres, puesto que aún no sabemos si es cubo. Y esto tentaremos por esta manera: sacaremos del número 5, nombre menor, la unidad, porque es cubo, y quedarán 4 , cuyo tercio es $11 / 3$, y si el dicho binomio es cubo y la división del número 5 es bien acertada, la raíz cuba de 1, que también es 1, será el nombre menor de la raíz cuba binómica que buscamos (Núñez 1567: fol. 336v-337r).

\subsection{Binómino}

Este sustantivo, sinónimo de binomio $-\mathrm{y}$ con apariencia de variante gráfica-, proviene del latín BINŌMINIS, -E 'que tiene dos nombres', según el OLD, probablemente por analogía con binomio, esto es, mediante la adición del prefijo latino BI- 'dos veces' (DRAE) y el genitivo singular NŌMIINIS, de la forma lat. NŌMEN, -INNIS 'nombre' (DECH).

Este término algebraico se documenta en 281 ocurrencias en el corpus del DICTER, únicamente en el primer libro de Álgebra publicado en España, esto es, en la Arithmética algebrática del alemán Marco Aurel, quien hace referencia al Libro X de los Elementos de Euclides ${ }^{17}$ y a la cita a la que el TLFi expone como la traducción latina del

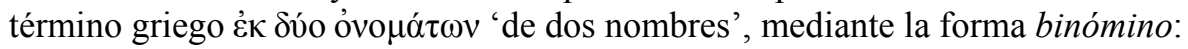

Trata de binóminos y residuos, y de sus definiciones, declaración y operación:

Euclides en la 30 del $10^{\circ}$ demuestra, diziendo: «Si dos líneas, que solamente en potencia serán racionales comunicantes, fueren juntadas in longum, digo que si d'estas dos líneas fuere hecha una, toda esta línea assí compuesta será irracional, llamada binómino».

D’estas líneas o binóminos ay 6, porque en 6 maneras y no en más, podrá venir tal ajuntamento. Quiero ponerlas aquí a la larga, para que las entiendas, pues para el $10^{\circ}$ de Euclides hazen muy mucho al caso, y no menos para muchas otras demandas que a las manos te podrán venir. Y primero porné la definición de cada uno y luego sus declaraciones (Aurel 1552: fol. 55v).

${ }^{17}$ Ejemplo en el que se pone de manifiesto la relación existente entre álgebra y geometría. Detalle fundamental, ya que la confluencia entre ambas disciplinas era para muchos autores de esta centuria, entre ellos Núñez, indisoluble; de hecho el matemático portugués llegó a afirmar que: «quien sabe por Álgebra sabe scientíficamente. Principalmente, que vemos algunas vezes no poder un gran mathemático resolver una questión por medios geométricos y resolverla por Álgebra, siendo la misma Álgebra sacada de la Geometría, que es cosa de admiración» (Núñez 1567: fol. 268v), aquí el autor aporta la idea de «relacionar el coneixement de l'àlgebra amb el coneixement científic, hom pot pensar Aristotèlic. Àlgebra com a ciència que permet demostrar un problema que geomètricament un altre matemàtic no ha pogut resoldre» (Massa Esteve 2010: 118). 
Un detalle interesante es el hecho de que este término exista en lengua portuguesa (cf. binòmine, DLP) y, sin embargo, no aparezca documentado en el Libro de Álgebra de Núñez, que se decanta por las formas en -nomio ${ }^{18}$.

A pesar de que exista una diferencia formal con respecto a binomio, creemos que se trata de dos expresiones sinónimas, referidas a términos algebraicos que constan de 'dos nombres' o 'dos números', ya que en los textos matemáticos del siglo XVI se emplea a menudo el vocablo nombre para referirse a número (Molina Sangüesa 2015b), es decir, a la «expresión de una cantidad con relación a su unidad» (DRAE: s. v. número) ${ }^{19}$.

En esta línea se explican las definiciones empleadas por el llamado Diccionario de Autoridades: «Term. de Aritmética. El agregado de dos números inconmesurables, de que trata Euclides, y pone seis especies en el libro décimo de sus elementos» o del Diccionario castellano de Esteban de Terreros y Pando: «Término del Álgebra, es el número producido de la suma de dos números o magnitudes inconmensurables. Cuando hay tres magnitudes se llama trinomio, si hay cuatro cuatrinomio, y si más multinomio» ${ }^{20}$. No obstante, consideramos más apropiada la definición del DRAE, el cual indica que un binomio es una «expresión compuesta de dos términos algebraicos unidos por los signos más o menos». No hay duda de que el étimo, en ambos casos, binomio y binómino, y por analogía en el resto de la serie (trinomio/ trinómino, etc.) proviene, respectivamente, del latín NŌMEN, -ǏNIS y del francés NÔME, y no del griego vouó $\varsigma$ 'parte, porción' como considera el DRAE en sus sucesivas ediciones, entre otros repertorios lexicográficos.

\subsubsection{Binominal}

Sinónimo de binómico, este adjetivo relacional derivado de binómino (del lat. BINOMǏNE 'que tem dois nomes' $+-a l^{21}$, según DLP) aparece en 12 ocurrencias en nuestro corpus y se refiere a aquello «perteneciente o relativo al binomio» (DRAE: $s . v$. binomial), tal y como se comprueba en los siguientes ejemplos extraídos de las obras de Marco Aurel y Pérez de Moya, donde se percibe una fuerte influencia ejercida por el matemático alemán:

La causa muy necessaria d'esta tal multiplicación del binómino con su residuo, o residuo con su binómino, es porque venga sola una quantidad o número discreto a ser tu partidor. Porque, siendo el partidor binominal, sería impossible por él partir quantidad alguna; mas también se multiplica la summa partidera con la mesma quantidad con la que fue multiplicado el partidor, porque no cresce ni mengua la

${ }^{18}$ En línea con la corriente italiana del pensamiento matemático, de la cual era un buen conocedor (Molina Sangüesa 2015a).

${ }^{19}$ Una muestra de ello es este fragmento de la Aritmética del matemático catalán Juan de Ortega: «Busca un nonbre que, tomando la mitad d'él ayuntada con los 6 que tenía el menor, que monten 20, el qual nombre será 28, porque su mitad son 14» (1512: fol. 189v).

${ }^{20} \mathrm{Si}$ no se indica lo contrario en la bibliografía final, las referencias a los diccionarios están tomadas del NTLLE de la Real Academia Española.

21 «Sufijo comunísimo que sirve para derivar adjetivos a partir de bases nominales. Se remonta a -ālis, sufijo latino que tiene la misma función gramatical [...] en numerosos latinismos o derivados sirve para expresar pertenencia u otra relación» (Pharies 2002: 57-59). 
proporción quando los dos números son multiplicados por una común quantidad (Aurel 1552: fol. 60v).

Nota. Alguno podría dezir: ¿para qué se multiplica quando el partidor es residuo por su binomio, y al contrario, si es binomio, por su residuo? A esto se responde que por reduzir, o hazer que sea el partidor sola una quantidad, porque siendo el partidor binominal será impossible poder partir con él ninguna quantidad (Pérez de Moya 1562: 541-542).

\subsection{Otras expresiones algebraicas}

Como a continuación comprobaremos, por analogía con binomio y binómino se crea toda una serie o paradigma de expresiones algebraicas formadas por más de dos términos.

\subsubsection{Trinomio y trinómino}

Ambos términos son sinónimos, se refieren a la «expresión algebraica compuesta de tres términos unidos por los signos más o menos» (DRAE: $s . v$.).

Trinomio, del mismo modo que binomio, está compuesto, según el diccionario académico — desde su edición de 1884 - por tri- y el gr. vó $\mu$ os 'partición' (DRAE). Sin embargo, en las ediciones anteriores (la de 1817 y 1780, concretamente) y en el Diccionario de Autoridades se documenta el étimo latino TRINOMIUM.

También en el Laterculi vocum latinarum se registra la forma latina TRINOMIUS, la cual consideramos que se creó por analogía con BINOMIUS, a partir del prefijo cuantificador TRI- 'tres' y el latín NŌMEN 'nombre', esto es, 'de tres nombres' o a partir de TRIy del fr. NÔME (TLFi), de acuerdo con la interpretación que presenta el diccionario galo $^{22}$ y los ejemplos del corpus del DICTER ${ }^{23}$ :

Queriendo sacar r. de algún trinomio, assí como de 9cce. p. 12cu. p. 4ce., sacarás la r. de los extremos, y si la multiplicación de las raýzes de los dos extremos hiziere tanto como la mitad del charácter de en medio de los 3 que quisieres sacar r., el tal trinomio tendrá r., y la tal r. es la misma r. de los extremos (Pérez de Moya 1562: 523).

Todo binomio de dignidades, multiplicado en sí, haze trinomio, porque las quadraturas no se juntan sino por esta palabra más o menos, mas no responde a qualquier trinomio de dignidades raíz que sea binomio. Por lo qual, para que sepamos si el trinomio que nos proponen tiene raíz que sea binomio, avemos primeramente de ver si en el trinomio ay dos dignidades quadradas, porque, si no las ay, no podrá ese trinomio tener binomio por raíz. Y puesto que tenga dos dignidades quadradas, aun eso no bastará para que tenga raíz que sea binomio, porque juntamente con esto conviene que las raízes de las dos dignidades quadradas sean tales, que una, multiplicada por otra, haga la mitad de la tercera dignidad del trinomio (Núñez 1567: fol. 131r-131v).

Sin embargo, en otros repertorios lexicográficos hallamos una interpretación contrapuesta: la que defiende el $\mathrm{DRAE}^{22}$ y que propone una etimología proveniente del

${ }^{22}$ Documentado en 1554 (J. Peletier Dumans, L'Algèbre, second livre, p. 183), el TLFi afirma que este polinomio de tres términos (trinôme, en francés) es «formé des élém. formants tri-* et -nôme* d'apr. binôme». A diferencia de binomio, este sustantivo no aparece recogido en los diccionarios etimológicos italianos de Manlio Cortelazzo ni de Niccolò Tommaseo.

${ }^{23}$ Se documenta 65 ocasiones en los tratados de Marco Aurel y Juan Pérez de Moya. 
griego vó $\mu$ os. Entre otros, el VME de Picatoste, que expone que trinomio está tomado «del gr. TRIA, 'tres' y NOME, 'parte'», o los diccionarios lusos, como el compuesto por Figueiredo: «do gr. TREIS + NOMOS» y el DLP: «do grego TREĨs, 'três' + NÓMOS, 'divisão' + -io».

Por otro lado, el sustantivo trinómino, que aparece en 15 ocasiones en el Libro primero de Arithmética algebrática de Aurel, no se documenta en ninguno de los repertorios lexicográficos analizados. Lo más próximo es trinómine, registrado en DLP con marcación diatécnica «poético» y como un término tomado «do latim TRINOMINNIS, -E 'idem'», el cual, por analogía con el latino BINOMINIS, se formaría mediante la adición del prefijo numeral TRI- 'tres' y el genitivo singular NŌMĬNIS, de la forma latina NŌMEN, -INNIS 'nombre' $(\mathrm{DECH})$. Al mismo tiempo, se nos remite a trinominal, — término también recogido en la obra del matemático alemán-, adjetivo relacional que se define como «que tem três nomes» (DLP: s. v. trinominal):

Y con este trinómino multiplica el partidor primero, y verná número discreto a ser tu partidor, con el qual partirás la suma que querrás partir, haviéndola primero multiplicado con el trinómino con el qual multiplicaste el partidor primero, por la mesma susodicha $17^{\mathrm{a}}$ del $7^{\circ}$, y como en las otras particiones de binóminos has visto (Aurel 1552: fol. 62r).

Agora, multiplica la summa partidera, que es 60 , por el mesmo trinómino, y vernán rrr. de $5400000+$ rrr. de $2160000+$ rrr. de 864000 . Éstas parte por 3 (reduzido a cubo), y verná el quociente a ser rrr. de $200000+$ rrr. de $80000+$ rrr. de 32000 . Y si los querrás provar, multiplica el quociente trinominal con el partidor primero, que es rrr. de 5 - rrr. de 2, y vernán los 60, etc. (Aurel 1552: fol. 62v).

\subsubsection{Cuadrinomio y cuatrinómino}

Este par de sinónimos se definen, del mismo modo que sus predecesores, como una «expresión algebraica compuesta de cuatro términos unidos por los signos más o menos». Por lo general, salvo cuadrinomio que aparece, aunque sin precisar etimología, en el DRAE ${ }^{22}$, no se documentan en los repertorios lexicográficos consultados.

Por un lado, consideramos que cuadrinomio se trata, al igual que trinomio, de un compuesto formado por el lat. QUADRI- 'cuatro' (DRAE) y el fr. NÔME 'de cuatro nombres' por analogía con binomio (TLFi). Expresión algebraica que se documenta en 14 ocasiones en el corpus distribuidas en las obra de Pérez de Moya y Núñez Salaciense con la variante gráfica quadrinomio:

La qual r.60 doblarás multiplicando por 4 (como se mostró en el tercero aviso, artículo sexto del quarto capítulo) y montará r.240, lo qual summarás con los 20 , que es la summa de las potencias de las dos partes en que dividiste esta quantidad o quadrinomio, y será todo 20 p. r.240, como hemos dicho (Pérez de Moya 1562: 533).

Algunas vezes, en la resolución de algunos qüesitos, viene la ygualación a parar en quadrinomio de dignidades ygual a número, por lo qual sería necessario buscarle raíz para que la raíz del número quedasse ygual a la raíz del quadrinomio, pero ningún quadrinomio de dignidades tiene raíz, excepta la universal, la qual no nos podrá servir, porque binomio de dignidades, siendo en sí multiplicado, haze trinomio, como avemos dicho, y no quadrinomio (Núñez 1567: fol. 134v). 
Sin embargo, por otro lado, documentamos 5 ocurrencias en la obra del alemán Marco Aurel la forma quatrinómino, que presenta un prefijo numeral distinto: el alomorfo cuatri-, elemento compositivo del término formado por el lat. QUATRI- 'cuatro' $\left(\mathrm{DRAE}^{22}\right.$ ) y del lat. NŌMEN, -INNIS 'nombre' (DECH), por analogía con binómino:

Nota. Quando te verná algún binómino, trinómino, quatrinómino, etc., mira si ay algunas quantidades que sean comunicantes, que las puedas summar, de tal manera que del quatrinómino puedas hazer trinómino o binómino; o del trinómino, binómino, o raýz de número no quadrado; o del binómino hazer raýz de número no quadrado (Aurel 1552: fol. 54r).

Agora, multiplica la summa partidera, que es 8 , con rr. de $6-\sqrt{2}$, que es el residuo del primer partidor, y vernán rr. de $24576-\sqrt{128}$, los quales torna a multiplicar con el binómino del segundo partidor, que es $\sqrt{6}+2$, y vernán rr. $884736-\sqrt{768}+$ rr. de $3932263-\sqrt{ } 512$. Todo este quatrinómino partirás por el 2 , que es tu partidor racional y común para todos estos quatro números del dicho quatrinómino (Aurel 1552: fol. 61v).

\subsubsection{Quinomio}

Este sustantivo que indica una «expresión algebraica compuesta de cinco términos unidos por los signos más o menos», no aparece recogido en ninguno de los diccionarios examinados. No obstante, en el corpus del DICTER se documenta una ocasión en la Arithmética práctica de Pérez de Moya y 15 en el Libro de Álgebra de Núñez Salaciense:

Si quisieres sacar r. de 16 cecu. p. 24 R. p. $25 \mathrm{cce}$ p. 12 cu. p. 4 ce., sacarás, como arriba, r. de los dos extremos, y será 4cu y 2 co. Aora, si este quinomio tiene r., tanto vendrá partiendo el segundo charácter, que es 24R., por la r. del primero extremo, que es 4 cu., como partiendo el quarto charácter, que es $12 \mathrm{cu}$., por la r. del último, que es 2co., que a qualquiera d'estas particiones salen 6ce. (Pérez de Moya 1562: 523-524).

Y si quisiéssemos tomar por raíz un trinomio, ny aun eso podrá ser, porque trinomio es raíz de quinomio y no de quadrinomio, como se muestra por sus multiplicaciones, $\mathrm{y}$, por tanto, daremos la unidad al quadrinomio de dignidades para que resulte quinomio, y daremos también la unidad al número que se propone ygual al quadrinomio para resultaren yguales un quinomio y el número, y entonces podremos buscar raíz del constituido quinomio, que no sea universal, y, si la tuviere, será trinomio de dos dignidades con la unidad (Núñez 1567: fol. 134v).

$\mathrm{Al}$ igual que el resto de las formas en -nomio analizadas, consideramos que este término se trata de un compuesto formado del latín QUĪNQUE 'cinco' (DECH) y el francés NÔME 'de cinco nombres' por analogía con binomio (TLFi).

Del mismo modo que los sustantivos cuadrinomio y cuatrinomio analizados, no presenta ningún derivado en -ico o - al.

\subsubsection{Senomio}

A continuación, hallamos el nombre que responde a la «expresión algebraica compuesta de seis términos unidos por los signos más o menos», esto es, un senomio. Sustantivo que, en nuestra opinión, es un compuesto del latín SEX 'seis' (DECH) y el francés NÔME 'de seis nombres', por analogía con binomio (TLFi). 
A pesar de que este término, al igual que sucede con quinomio, no se registre en ninguno de los diccionarios examinados, aparece testimoniado en una ocasión en el corpus que manejamos, en la obra del matemático portugués:

Y porque aún raíz de 60 más 2 es quantidad compuesta, multiplicaremos raíz de 60 más 2 por raíz de 60 menos 2 , y haremos 60 menos 4 , que son 56 , que será partidor simple. Y multiplicaremos también raíz de 30.000 más raíz de 50.000 menos raíz de 60.000 por raíz de 60 menos 2, y haremos este senomio: raíz de 1.800 .000 más raíz de 3.000 .000 más raíz de 240.000 menos raíz de 3600000 menos raíz de 200.000 , a partir por 56, y verná el mismo quociente que vernía partiendo 100 por raíz de 3 más raíz de 5 más raíz de 6 (Núnez 1567: fols. 63r-63v).

\subsubsection{Multinomio}

Finalmente, destacamos el término multinomio, documentado un par de ocasiones en el Libro de Álgebra de Pedro Núñez, cuyo significado es la «expresión compuesta de dos o más términos algebraicos unidos por los signos más o menos» (DRAE: $s . v$. polinomio), término que englobaría al resto de las expresiones algebraicas anteriormente analizadas:

Y la misma arte nos podrá servir para partir número o raíz simple por trinomio, quadrinomio, o qualquier otro multinomio, porque iremos mudando el más en menos y el menos en más tantas vezes quantas cumpliere, y haremos tantas multiplicaciones hasta que paremos en partidor simple (Núñez 1567: fol. 62v- 63r).

El mismo modo ternemos quando el partidor fuere trinomio, o quadrinomio o qualquier otro multinomio de raízes quadradas. Pero sabe que, assí como el partidor se va haziendo simple, la quantidad que avemos de partir se va haziendo más compuesta por esta arte de reduzir (Núñez 1567: fol. 64r).

Uno de los detalles más llamativos es el empleo de este vocablo: multinomio, formado por el elemento compositivo de origen latino MULTI- 'muchos' (DRAE) y el francés NÔME por analogía con binomio (TLFi), en lugar del término polinomio del que es sinónimo y que prevalece o conservamos en la actualidad.

Multinomio aparece recogido en el VME de Picatoste, quien remite a polinomio en su definición (1862: 78). De manera análoga, en el diccionario del filólogo portugués Cândido de Figueiredo se documenta el término multinómio, aunque no aporta etimología y se define como «o mesmo que polinómio ${ }^{24} »$.

Por último, cabe destacar que tampoco se recoge en los tratados matemáticos del corpus manejado el término monomio que hace referencia a la «expresión algebraica que consta de un solo término», según el DRAE.

\section{CONCLUSIONES}

Como adelantábamos en el análisis, las conclusiones que hemos obtenido tras haber revisado los términos referidos al paradigma de las expresiones algebraicas presentes en textos matemáticos del siglo XVI, ponen de manifiesto que, frente a la propuesta más extendida y generalizada entre los lexicógrafos — que postulan una etimología de ori-

${ }^{24}$ Cuya etimología (gr. POLUS + NOMOS), al igual que la de binomio, alude al término griego 
gen heleno: vouós 'parte'-, nos inclinamos por considerar más apropiada o acertada la que proviene del genitivo latino NŌMǏNIS (del latín NŌMEN, -ĬNIS 'nombre', para las formas en -nómino) y del francés NÔME (del mismo étimo latino: NŌMEN, -ĬNIS, en el caso de los compuestos en -nomio), de acuerdo con el DECH y TLFi.

En cuanto a su empleo por parte de los autores de tratados matemáticos en el Renacimiento español, podemos observar que es Núñez Salaciense el que ofrece una clasificación más exhaustiva, al examinar en su Libro de Álgebra la serie compuesta por las expresiones: binomio, trinomio, cuadrinomio, quinomio, senomio y multinomio - siendo estas dos últimas expresiones algebraicas únicamente tratadas por el portugués-. Por otro lado, Pérez de Moya, el más claro y pedagógico en sus exposiciones, da una pista sobre la posible etimología, que coincide con la tesis expuesta y defendida en este trabajo. Finalmente, Aurel, el introductor el Álgebra en España, quien, al parecer, intenta adaptar esta nueva terminología a la lengua vulgar, presenta expresiones más próximas a la forma latina, lo que configura toda una serie de expresiones algebraicas en -nómino: binómino, trinómino, cuatrinómino, e incluso un derivado, binominal, hoy extintas.

Las innovaciones de Aurel, que probablemente se debieran al desconocimiento de las formas españolas tradicionales, y su intento por adaptar al español este léxico de especialidad provocan, en el Quinientos, la convivencia de una dualidad de términos: binomio y binómino y, en consecuencia, todo un paradigma de expresiones algebraicas que alterna los nombres en -nomio y -nómino estudiados.

En definitiva, consideramos que esta revisión puede arrojar alguna luz sobre uno de los conceptos poco estudiados y no siempre bien interpretados en la historia de la lengua española, que es el de la terminología matemática relativa al Álgebra.

\section{REFERENCIAS BIBLIOGRÁFICAS}

\section{Fuentes primarias}

AUREL, Marco (1552): Libro primero de Arithmética algebrática, Valencia, Joán de Mey.

EUCLIDES (1899): Anaritii in decem libros priores elementorum Euclidis commentarii, ed. y trad. de Ernst Ludwig Wilhelm Maximilian Curtze, Leipzig, B. G. Teubneri.

(1991-1996): Elementos, Libros I-XIII, ed. y trad. de Ma Luisa Puertas Castaños, Madrid, Gredos.

Molina CANO, Joán Alfonso de (1598): Descubrimientos geométricos, Anvers, Andrea Bacx.

NúÑEZ, Pedro (1567): Libro de Álgebra en Arithmética y Geometría, Anvers, Herederos de Arnoldo Birckman.

ORTEGA, Juan de (1512): Conpusición de la arte de la Arismética y de Geometría, León de Francia, Maistro Nicolau de Benedictis (por Joannes Trinxer).

PÉREZ DE MOYA, Juan (1562): Arithmética práctica y speculativa, Salamanca, Mathías Gast.

Estudios y repertorios lexicográficos

DCVB: AlCOVER, Antoni y Francesc de Borja Moll (1927): Diccionari català-valenciàbalear, Palma de Mallorca, Imprenta de A. M. Alcover. 
DLI: TOMmaseo Niccolò (1861-1879): Dizionario della Lingua Italiana [DLI]. [En línea]: $<$ http://www.dizionario.org/> [Consulta: 21/10/2013].

BoncompAGNI, Baldassare (1851): «Della vita e delle opere di Gherardo Cremonese, traduttore del secolo decimosecondo, e di Gherardo da Sabbioneta, astronomo del secolo decimoterzo», Atti dell'Accademia Pontificia dei Nuovi Lincei, IV, pp. 387-493.

DELI: CoRTelazzo, Manlio y Paolo Zoli (1979-1988): Dizionario etimologico della lingua italiana, Bologna, Zanichelli.

DECH: Corominas, Joan y José Antonio PASCuAl (1980-1991): Diccionario crítico etimológico castellano e hispánico, Madrid, Gredos.

DICTER: MANCHO DuQue, Mª Jesús, dir. (2014): Diccionario de la ciencia y de la técnica del Renacimiento. [En línea]: <http://dicter.eusal.es> [Consulta: 5/01/2014].

DLP: Diccionário da Língua Portuguesa. [En línea] Infopédia, Enciclopédia e DicionáriosPortoEdito-

$\mathrm{ra}:<\mathrm{http}: / /$ www.portoeditora.pt/espacolinguaportuguesa/dol/dicionarios-online/ $>$ [Consulta: 20/06/2013].

DRAE: REAL ACADEMIA ESPAÑOLA $\left(2001^{22}\right)$ : Diccionario de la lengua española, Madrid, Espasa Calpe. [En línea]: <http://buscon.rae.es/diccionario/drae.htm $>$ [Consulta: 5/07/2013].

FlóReZ Miguel, Cirilo (2006): «Ciencias, siglos XV-XVII», en Luis E. Rodríguez-San Pedro Bezares, coord., Historia de la Universidad de Salamanca, III: Saberes y confluencias, Salamanca, Universidad de Salamanca, pp. 409-433.

GAFFIOT, Félix (1934): Dictionnaire latin-français, París, Hachette.

Gradenwitz, Otto (1904): Laterculi Vocum latinarum, Leipzig, Von S. Hirzel.

LEAL Y LEAL, Luis (1971-1972): «El Bachiller Juan Pérez de Moya», Boletín del Instituto de Estudios Giennenses, 70-71, pp. 17-36.

MANCHO DuQue, M. ${ }^{a}$ Jesús, dir. y Mario QuiRós GARCíA, coord. (2005): La ciencia y la técnica en la época de Cervantes: textos e imágenes, Salamanca, Universidad de Salamanca.

Massa Esteve, M. ${ }^{a}$ Rosa (2010): «Àlgebra i Geometría al Libro de Álgebra en Arithmética y Geometría (1567) de Pedro Núñez», Quaderns d'Història de l'Enginyeria, XI, pp. 101-129.

MOLINA SANGÜESA, Itziar (2015a): «Tradición e innovación en el ámbito de la divulgación matemática de Quinientos», en Jenny Blume y Carmen López Ferrero, eds., La ciencia como diálogo entre teorías, textos y lenguas, Berlín, Frank \& Timme, pp. 31-48.

(2015b): Las matemáticas en el Renacimiento hispano: estudio léxico y glosario. Salamanca: Universidad de Salamanca [Tesis doctoral inédita].

NDLP: FigueIREDO, Cândido (1899): Novo Dicionário da Língua Portuguesa.[En línea] $<$ http://www.gutenberg.org/cache/epub/31552/pg31552.html $>$ [Consulta: 20/06/2013].

NTLlE: ReAl ACADEMia Española (2001): Nuevo Tesoro Lexicográfico de la Lengua Española (edición DVD), Madrid, Espasa Calpe.

OLD: Glare, P. G. W. (1968-1982): Oxford Latin Dictionary, Oxford, Clarendon Press.

PARADís, Jaume y Antoni MALET (1989): Los orígenes del álgebra: de los árabes al Renacimiento, Barcelona, PPU.

PHARIES, David (2002): Diccionario etimológico de los sufijos españoles, Madrid, Gredos.

PICATOSTE Y RODRÍGUEZ, Felipe (1891): Apuntes para una biblioteca cientifica española del siglo XVI, Madrid, Imprenta y Fundación Manuel Tello. 
Real ACAdemia Española (1726-1739 [1990]): Diccionario de Autoridades, Madrid, Gredos.

- : Mapa de diccionarios académicos. [En línea]: <http://web.frl.es/ntllet/> [Consulta: $25 / 03 / 2013]$.

REY PASTOR, Julio (1926): Los matemáticos españoles del siglo XVI, Madrid, Biblioteca Scientia.

TERREROS Y PANDO, Esteban de (1786-1793 [1987]): Diccionario castellano con las voces de las ciencias y las artes, Madrid, Arco/Libros.

TLFi: Trésor de la Langue Française informatisé, París, CNRS éditions [En línea]: $<$ http://atilf.atilf.fr/tlf.html $>$ [Consulta: 18/10/2013].

Valladares Reguero, Aurelio (1997): «El Bachiller Juan Pérez de Moya: Apuntes biobibliográficos», Boletín del Instituto de Estudios Giennenses, 165, pp. 371-412.

Ventura Sousa, Manuel (1985): Vida e Obra de Pedro Nunes, Biblioteca Breve, 99, Lisboa, Instituto de Cultura e Língua Portuguesa, Ministério da Educação.

VERNET GINÉS, Juan (1978): La cultura hispanoárabe en Oriente y Occidente, Barcelona, Ariel.

(2006 $\left.{ }^{2}\right)$ : Lo que Europa debe al Islam de España, Barcelona, Acantilado.

VLI: Bruno MigLIORINI (1965): Vocabolario della Lingua Italiana Torino, Paravia.

VME: PiCATOSTE Y RodRígueZ, Felipe (1862): Vocabulario matemático-etimológico, Madrid, Imprenta y Librería de D. E. Aguado. 